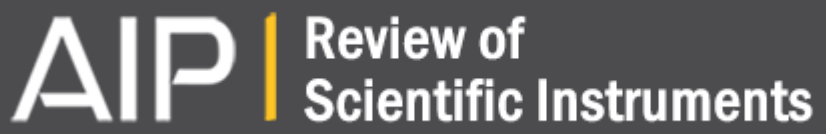

Superiority of a Knudsen Type Vacuum Gauge for Large Metal Systems with Organic Vapor Pumps; Its Design and Operation

Jesse W. M. DuMond and W. M. Pickels Jr.

Citation: Review of Scientific Instruments 6, 362 (1935); doi: 10.1063/1.1751900

View online: http://dx.doi.org/10.1063/1.1751900

View Table of Contents: http://scitation.aip.org/content/aip/journal/rsi/6/11 ?ver=pdfcov

Published by the AIP Publishing

\section{Articles you may be interested in}

Design and operation of scroll-type dry primary vacuum pumps

J. Vac. Sci. Technol. A 19, 1708 (2001); 10.1116/1.1359543

Operation and maintenance of a diffusionpumped vacuum system

J. Vac. Sci. Technol. 16, 71 (1979); 10.1116/1.569870

Turbomolecular pump vacuum system for the Princeton Large Torus

J. Vac. Sci. Technol. 15, 734 (1978); 10.1116/1.569500

Calibration of a Knudsen Type Vacuum Gauge

Rev. Sci. Instrum. 36, 1658 (1965); 10.1063/1.1719426

Titanium Vapor Vacuum Pump and Bayard-Alpert Type Ionization Gauge Am. J. Phys. 30, v (1962); 10.1119/1.1942139

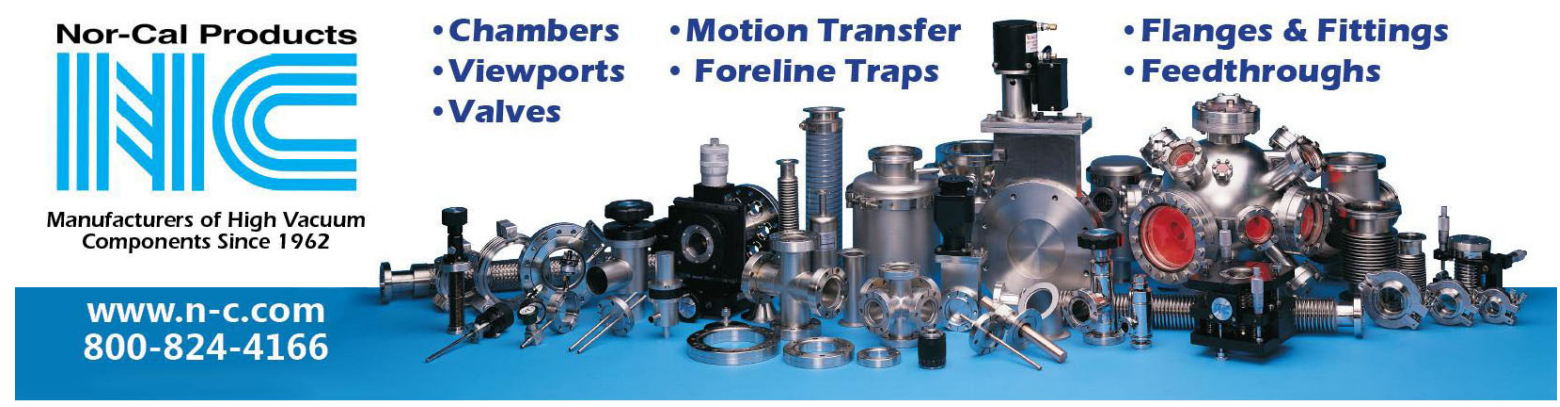




\title{
Superiority of a Knudsen Type Vacuum Gauge for Large Metal Systems with Organic Vapor Pumps; Its Design and Operation
}

\author{
Jesse W, M. DuMond and W. M. Pickels, JR., California Institute of Technology
}

(Received July 22, 1935)

\begin{abstract}
The McLeod, Pirani, Knudsen and ionization gauges have been studied as to their suitability in the operation of large metal vacuum systems with organic vapor pumps now coming into extensive use in modern physics. Advantages and disadvantages of each type based on our experience are critically discussed. The superiority of a gauge on the Knudsen principle for this work is stressed and we present a new design and a simpler mode of operation of such a gauge together with a full discussion of its construction, calibration and characteristics.
\end{abstract}

A $\mathrm{N}$ entire techinique of "vacuum plumbing" in very large scale metal systems is now well advanced as a result of the requirements of recent new techniques ${ }^{1}$ in modern physics and especially nuclear physics. Such systems usually are provided with organic vapor pumps containing brass and copper parts and using butyl phthalate or oils such as Apiezon. The authors have been occupied for some time with the construction of a large $\mathrm{x}$-ray tube designed for a continuous power of $30 \mathrm{kw}$ together with its constant voltage power supply system involving two large thermionic diode valves. Both $\mathrm{x}$-ray tubes and valve tubes are maintained at high vacuum by continuous pumping with two stage brass Apiezon oil vapor pumps.

\section{Reguirements and Discussion of Various Types of Gauges}

For such work we have in common with other workers found that a rough vacuum gauge is almost indispensable both in hunting for leaks in the metal systems and in studying and controlling the behavior of the vapor pumps under various

\footnotetext{
${ }^{1}$ Probably the first example of such a large system was the million-volt $x$-ray tube of $C$. C. Lauritsen at the California Institute. The "cyclatron" and the high frequency $x$-ray tube of Lawrence and his collaborators at Berkeley, the high voltage $x$-ray tubes of W. D. Coolidge, the large ion accelerating tubes of Tuve at the Bureau of Terresterial Magnetism, of Lauritsen and Crane at Pasadena and of Cockcroft and Walton at Cambridge are all excellent examples of the trend. Huge vacuum systems for spectroscopy have been constructed at Massachusetts Institute of Technology. Probably the largest high vacuum system in point of volume is that of John Strong used at California Institute of Technology for coating the 100-inch Mount Wilson Observatory Reflector.
}

operating conditions. A high precision is not essential for such a purpose but the gauge should perform reliably and reproducibly. It is convenient to have enough precision to detect a decided change in a slow rate of leak at pressures say from $10^{-6}$ to $10^{-5} \mathrm{~mm} \mathrm{Hg}$ with the pumps shut off. The gauges most commonly in use for such purposes are the McLeod, the ionization gauge and the Pirani gauge. We have tried all three of these and found each of them to have certain objectionable features as follows: The McLeod is quite reliable, simple and cheap but the mercury vapor is a most undesirable feature in connection with systems having brass and copper parts. The manufacturers of "Apiezon" oil emphatically advise against contamination of "Apiezon" oil pumps with mercury vapor if the highest ultimate vacuum is to be obtained, although there is some division of opinion among experimenters as to the seriousness of this harmful effect. The mercury vapor may be trapped off with a liquid-air trap placed between the gauge and the rest of the system but in the course of months of work the danger of neglecting to keep liquid air on the trap at all times is high and accidents or leaks may occur which actually carry even large quantities of mercury over into the main system. The McLeod does not give a continuous record of pressure but must be "read," an interval of a minute or more being required for the reading. The McLeod does not read the pressure of condensable vapors and these in large metal vacuum systems are decidedly a factor to be reckoned with.

The ionization gauge has high sensitivity and gives a continuous record of vacuum pressure which may be read at a very considerable 
distance from the gauge itself on a microammeter. Its disadvantages are principally due to the necessity for a hot filament and expensive auxiliary equipment and meters. The hot filament undoubtedly acts upon any organic vapors present in the gauge changing their molecular constitution or "cracking" them to a more permanent gas. Thus there is no assurance that the pressure read in the gauge is a true representation of the pressure in other parts of the system. Furthermore the filament is short lived and is a nuisance to replace. There is always danger of inadvertently lighting the filament before a sufficiently good vacuum has been reached to prevent burning it out. It is claimed by some that the ionization gauge need not be initially outgassed but when we did not take this precaution we found that a good fraction of the life of a filament would be expended waiting for the reading of the ionization gauge to reach a stable value and we always felt considerable doubt as to its reliability. Outgassing either with induction furnace or by thermionic bombardment is a tedious, complicated and time consuming operation. Finally an expensive sensitive microammeter is required and if no other source of direct current is available there must be both $\mathrm{A}$ and $\mathrm{B}$ batteries to be charged and replaced at intervals.

The Pirani gauge has nearly all the advantages and avoids almost all the disadvantages mentioned for the McLeod and ionization gauges. We have found, however, that it is not practicable to make it sufficiently sensitive and stable in the pressure range from $10^{-6}$ to $10^{-5} \mathrm{~mm} \mathrm{Hg}$. This statemenit requires some amplification. After a very thorough study of the Pirani gauge and much experimentation with different methods of operating and reading it our best result was obtained with a pair of gauges each member of which contained two filaments of about three meters of one-mil tungsten wire wound into helices on a ten-mil mandrel. Each of the helices when strung under tension in the gauge and supported only at the ends was about $20 \mathrm{~cm}$ long. One of the two gauges was pumped down and sealed off in connection with a well outgassed charcoal trap immersed in liquid air. This we called the reference gauge. The other gauge was connected to the vacuum to be measured. The four filaments in the two gauges whose resistances had been made as nearly equal as possible were connected to form the four arms of a Wheatstone bridge with only a very small variable resistance external to the gauges for balancing the bridge. The resistances in one and the same gauge were in opposite arms of the bridge. With this arrangement it was found easy to attain a sensitivity such that a change of pressure from zero to $10^{-4}$ $\mathrm{mm} \mathrm{Hg}$ would give several microamperes deflection on the bridge galvanometer and at this rate an ordinary wall galvanometer would theoretically serve very well to read pressures of from $10^{-5}$ to $10^{-6} \mathrm{~mm} \mathrm{Hg}$. The trouble was that the gauge proved to be as sensitive to nearly any accidental thermal or mechanical effect as it was to say $10^{-5} \mathrm{~mm} \mathrm{Hg}$ pressure. The envelopes of both reference and measuring gauges were brass cylinders provided with water jackets through which tap water flowed continuously. Nevertheless the bridge could be thrown out of balance by placing one's fingers on the water pipe leading to the jacket to a greater degree than $10^{-5} \mathrm{~mm} \mathrm{Hg}$ change in pressure would unbalance it. Tapping the gauge with the finger nail would do the same thing although the turns of the helix inside the gauge were amply far apart so that this could not have caused any of them to short circuit. As might be expected the balance of the Wheatstone bridge was unstable so that with perfectly constant vacuum conditions indicated on the calibrating McLeod the "zero" setting of the Wheatstone bridge would slowly drift from obscure unassignable causes many times as much as the amount corresponding to a change of pressure of $10^{-5} \mathrm{~mm} \mathrm{Hg}$. It was therefore quite possible to detect small and fairly rapid changes in pressure but in the absence of a McLeod it was quite hopeless to be sure of the absolute pressure itself. This drift of the zero was quite unsystematic and could not be blamed on voltage fluctuations in the battery supplying current to the bridge. To eliminate drift from this last cause we used a, D-D-51 Willard storage battery especially designed for maintaining very constant voltage. The drain of a milliampere or less on this battery was utterly negligible. A little calculation is sufficient to show that at about $3 \times 10^{-5} \mathrm{~mm} \mathrm{Hg}$ the loss of heat from the gauge wire by air conduction is only one percent of the loss by 
radiation for moderate temperature rises. Yet it is the change in resistance from the cooling effect of this one percent loss which must be measured in the presence of the other one hundred times greater loss by radiation. The slightest change in the emissivity of the surfaces of the wire or the envelope will therefore unbalance the bridge as much as the gas pressure to be measured. We are still at a loss to explain the sensitivity to mechanical vibration. No modification in the geometrical design of the gauge can improve the ratio of energy loss by gas conduction to energy loss by radiation since at these low pressures the atoms of gas travel in straight lines between the wire and the walls just as the photons of radiation do. Now this is the chief reason for the superiority of the Knudsen gauge. At these pressures the energy transported by molecules of gas is small compared to the energy transported by photons but the momentum transported by molecules is huge compared to the momentum transported by photons on account of the comparatively enormous mass of the atoms. The Pirani gauge measures the molecular energy transport while the Knudsen gauge measures the momentum transport, hence its superiority.

\section{The Knudsen vacuum gauge}

The Knudsen gauge consists essentially of a light suspended vane in a chamber communicating with the vacuum to be measured. One side of the vane is exposed to a source of heat at temperature $T_{1}$ while the other side is exposed merely to the walls of the chamber at temperature $T_{0}$. The "hot" gas molecules leaving the heater transport more momentum to one side of the vane than the cold molecules from the walls of the chamber transport to the other side and Knudsen has shown that in consequence the vane should experience a net displacing force per unit area given by

$$
F=(p / 2)\left[\left(T_{1} / T_{0}\right)^{1}-1\right] .
$$

Here $p$ is the gas pressure to be measured in the vacuum chamber, $T_{1}$ is the temperature of the heater and $T_{0}$ the temperature of the walls. The formula is seen to be independent of the molecular weight of the gas. The gauge will obviously cease to function correctly when the pressure has become so high and the mean free path conse- quently so short that convection currents are set up causing erratic behavior of the vane. We have under such conditions indeed observed that the vane instead of being repelled from the heater is attracted by it, probably because the rising convection current of hot gas between the vane and the heater produces a lowering of pressure by the Venturi effect. The theory of the gauge is based on the assumption that the mean free path of the gas molecules is long compared to the dimensions of the gauge.

In Dushman's excellent book High Vacua descriptions are given of three different Knudsen gauges built respectively by Knudsen, Woodrow, and Schrader and Sherwood and the fourth and latest by $\mathrm{H}$. Rieger is merely referred to under date 1920. From an examination of these articles we believe the reason why the Knudsen gauge in spite of its manifold advantages has been so completely shunned as a practical tool lies in the elaborate designs and precautions described by the above-named workers. The gauge itself was quite properly the object of a research problem to these men rather than a tool. They successfully extended its sensitivity to $5 \times 10^{-9} \mathrm{~mm} \mathrm{Hg}$ by

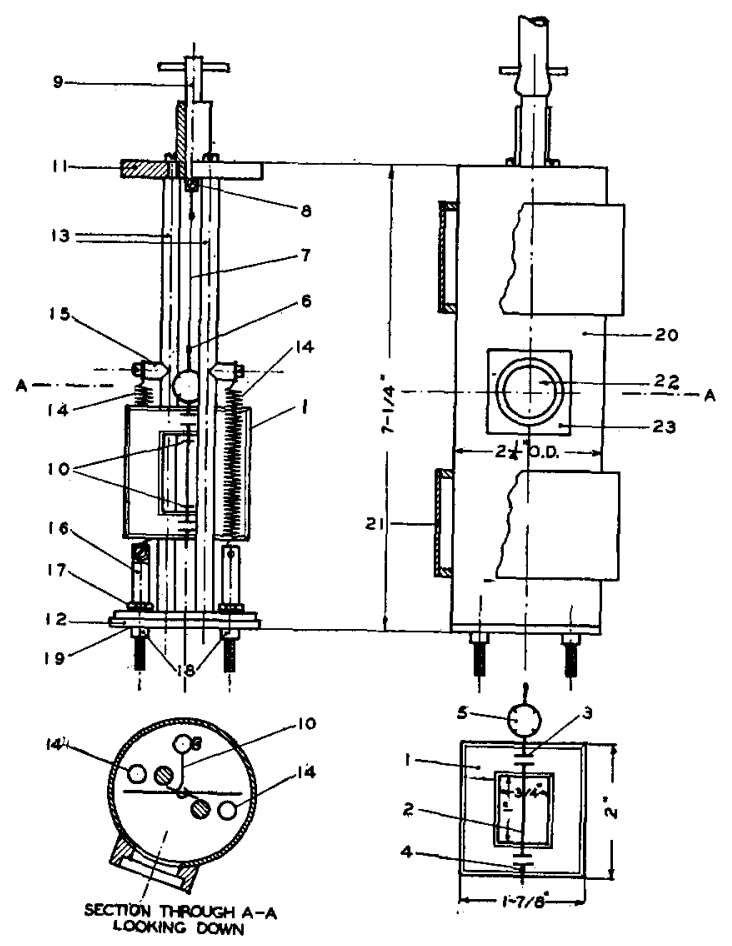

FIG. 1. Line drawing of Knudsen gauge. 


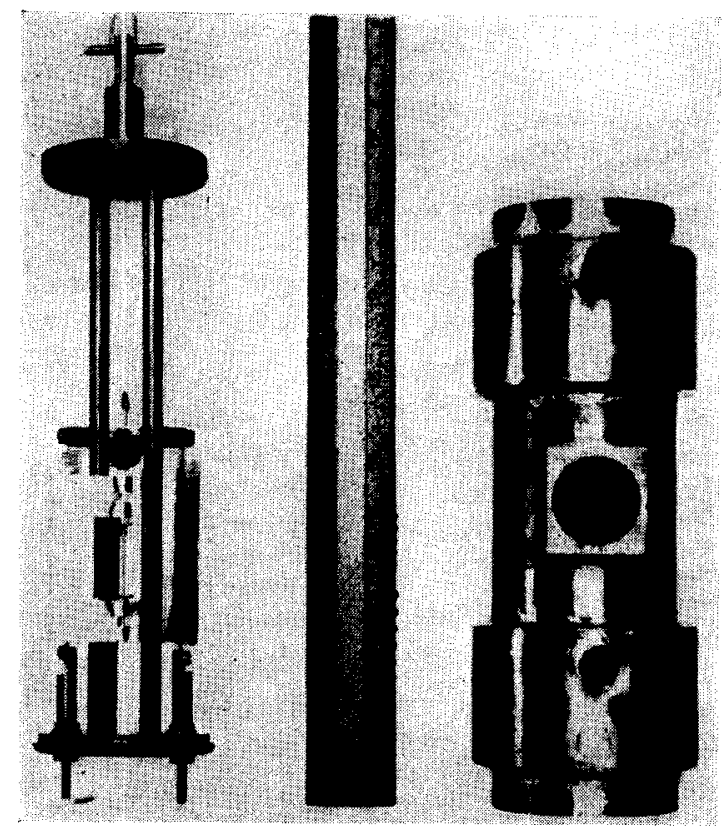

FIG. 2. Knudsen gauge disassembled.

careful outgassing. They compared its sensitivity with the theoretical formulae developed by Knudsen and to this end they provided elaborate means for measuring the temperature of the heater element, a flat platinum ribbon parallel to the movable vane. The gauges were built of glass and presented a fairly formidable job of glass blowing.

In the present instance our object was to develop a simple tool easy to construct and operate and suitable for the moderately good vacua of order from $10^{-5}$ to $10^{-6}$ with which we had to deal. There seemed no point in a glass gauge when the rest of the vacuum system is of metal and outgassing the gauge seems equally superfluous for these pressures when the gauge heater never operates at more than $70^{\circ} \mathrm{C}$ rise above the walls.

The advantages of the Knudsen gauge are that it involves no objectionable medium such as mercury with an undesirable vapor, it gives a continuous indication of pressure, it has no filament sufficiently hot to burn out or to change the chemical constitution of vapors or gases whose pressures are being measured, it is very stable and insensitive to external influences yet very sensitive to low pressures, its zero point can

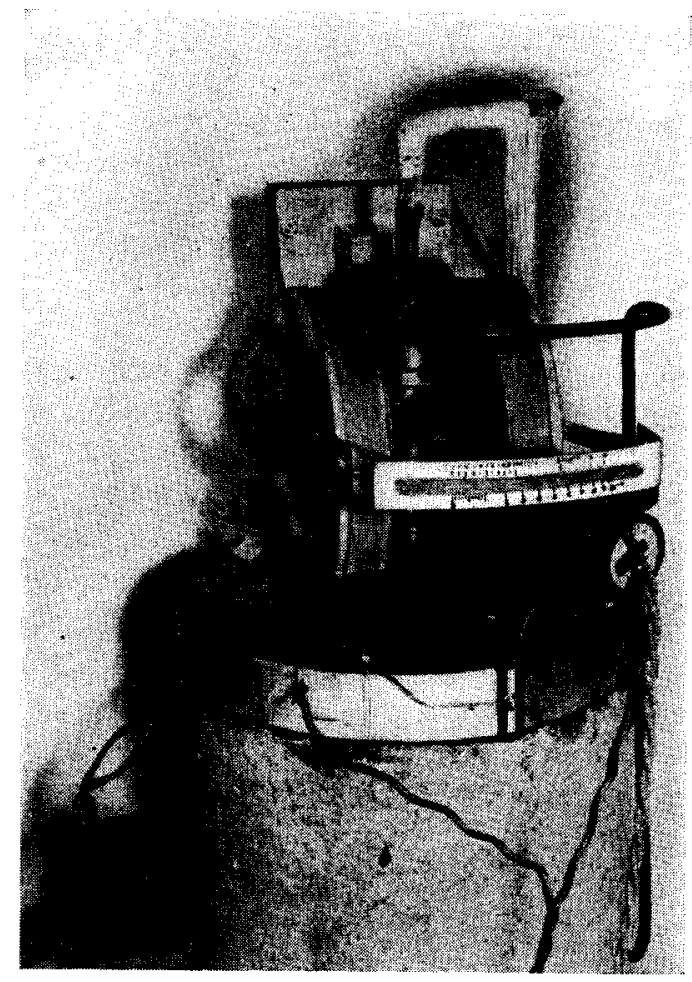

FIG. 3. General view of gauge. "mouse power" transformer appears at lower left.

always be checked by simply turning off the current to the heater, its use involves no expensive electrical measuring instruments, it measures the pressure of all vapors and gases alike quite independent of the masses of their molecules or their condensibility, and finally it is simple, easy and cheap to construct when built according to our design. It has but one minor disadvantage:-it must be mounted on a solid support fairly close to the vacuum system to be measured and the readings can only conveniently be taken by looking at the gauge scale itself.

Fig. 1 shows a cross section of the gauge while Figs. 2 and 3, respectively, are photographs of the inner part before the brass envelope has been soldered in place and of the assembled gauge with Helmholtz coils for damping. The movable vane (1), very like the one described by Schrader and Sherwood, consists of a rectangular frame of 2.5-mil sheet aluminum with both inner and outer edges folded over for stiffness. An axle (2) of 32-mil aluminum wire is threaded through slits (3) in this vane at the top and bottom and spot- 
welded lightly at one point (4) to prevent rotation. An ordinary galvanometer mirror, $1 \mathrm{~cm}$ in diameter (5) which can be purchased with an aluminum back support provided with ears for threading over the 32 -mil axle wire proved quite satisfactory since by dispensing with outgassing the mirror is not damaged. The top of the aluminum axle is bent into a small hook (6) round which the $\frac{1}{2}$-mil tungsten ( 7$)$ wire $(0.71 \mathrm{mg}$ per foot, to be precise) used for the suspension is wound about three times. The hook is then squeezed tightly shut with pliers and the wire never slips thereafter. This simple method we owe to Schrader and Sherwood. The other end of the suspension whose free length is about $5 \mathrm{~cm}$ is secured in the same way to a hook on a short length of aluminum wire which is then held by means of a set screw (8) in the hole in the bottom of the steel taper plug (9) which projects inside the gauge at the top. This steel plug made out of an ordinary machinist's dowel pin need only be a moderately good lapped fit in its brass taper socket which has been reamed with a standard taper reamer corresponding to the dowel. White lead is recommended for this lapping. The ultimate tightness is insured with a little soft laboratory wax or stopcock grease pressed in around the top of the plug. Some means of adjusting the equilibrium position of the vane after vacuum has been established in the gauge is absolutely essential because in the presence of air, convection currents make it quite impossible to make this adjustment. The taper plug with wax at the top may appear to be a crude solution but it is simple and entirely adequate and vacuum tight. Once adjusted it will never have to be touched again.

Since the gauge must be handled and turned about while soldering on the envelope two small eyes of 32-mil aluminum wire are provided through which the axle of the vane passes without contact when the gauge is properly leveled but which support the vane from swinging or breaking the suspensions when the gauge is being handled. These eyes are adjusted to the proper alignment and supported in holes with set screws in a vertical post provided for them. The brass disks (11), (12) forming the top and bottom of the gauge are held together before assembling the cylindrical brass envelope over them by two copper columns (13) screwed and soldered to the disks to insure vacuum tightness. Copper is used here for its good heat conductivity to minimize the time required for local temperature distributions to reach equilibrium. For the same reason the cylindrical outer envelope of the gauge is water jacketed above and below the mirror window. We have noticed a distinct improvement in reliability and promptness in reaching equilibrium when running water was used in these jackets although this is not absolutely essential.

The heaters (14) are simply helices of 60 inches of chromel resistance wire. No. 28 wound tightly on a $\frac{1}{4}$-inch mandel gives a helix which when extended has satisfactory uniformity of opening between turns and a resistance of $25 \mathrm{ohms}$. The heater helix must be so adjusted that the movable vane is prevented from short circuiting it by the copper post acting as a stop. The top of this helix is held by a set screw on a small copper arm (15) projecting from the side of the copper post. The bottom end is held by a set screw in the electrically insulated vacuum tight plug (16) passing through the brass disk which forms the bottom end of the gauge. These plugs are assembled with a nut (17) on the inside of the vacuum container and a shoulder (18) integral with the plug on the outside. The plug is insulated from the wall through which it passes with mica washers and a mica sleeve. It is rendered perfectly vacuum tight by painting the joint (19) on the outside of the gauge where the mica washer separates the shoulder on the plug from the wall against which it bears with a little "glyptal" lacquer. This ingenious bit of technique was originated by $\mathrm{C}$. C. Lauritsen and proves most convenient and satisfactory. We find it convenient though not indispensable to interpose a $\mathrm{U}$ trap for liquid air in the vacuum line leading from the gauge to the system to be measured for by means of this trap we can tell what part of the pressure measured by the gauge is due to condensable vapors. Since this trap should be of glass we have made the entire vacuum gauge line of glass connecting it to the metal gauge and to the metal system with small copper-to-"nonex" cylindrical seals which solder onto the metal parts conveniently. The cylindrical brass envelope (20) of the gauge with its window and 
water jackets (21) is simply slipped over the entire assembly and soft soldered to the disk shaped bottom and top end pieces once for all. The $\frac{3}{4}$-inch window (22) in this brass envelope consists of a small flat disk of plate glass 1 inch in diameter cemented in a suitable depression in a brass block (23) whose back side is shaped to fit the cylinder and soft soldered thereto. The glass is cemented to the brass with De Khotinsky cement. A swing of the light beam of about $30^{\circ}$ is permitted by this window.

Our gauge vane with the dimensions as shown has a period of about $5 \mathrm{sec}$. A little analysis will show that the magnetic field for critically damping a square loop of thin wire suspended like our vane is given by

$$
H^{2}=(26 / 3) \pi(\delta \rho / \tau) 10^{8}
$$

where $\tau$ is the period in seconds, $\delta$ the mass density of the wire and $\rho$ its electrical resistivity. This formula does not involve the dimensions of the loop save for the condition that the thickness of the wire must be small compared to the other dimensions. The formula gives about 60 gauss as the critical field for our case and this proved to be about right. The Helmholtz coils wound on wooden spools and clearly visible in the general view (Fig. 3) consist each of 512 turns of No. 20 cotton covered copper wire, the cross section of the total winding being $1^{\prime \prime} \times 1^{\prime \prime}$ and the mean diameter of the coil 7.1 inches. With the two coils in series 18 to 20 volts d.c. with no external resistance gives about the right field strength with only a very moderate temperature rise on the coils. With vacua better than $10^{-5} \mathrm{~mm} \mathrm{Hg}$ the magnetic damping is almost indispensable. The inductive "kick" received by the vane on making or breaking the current to the Helmholtz coils makes it preferable to leave the current running continuously while the gauge is in use. We have observed only the most negligible shifting of the "cold zero" position (heaters off) of the vane in consequence of the damping field and since our calibration of the Knudsen gauge against a McLeod was made with the magnetic field at its full strength a slight induced magnetic moment in the vane cannot falsify the deflections.

A small "mouse power" transformer visible at lower left, Fig. 3, with primary directly connected to the 110 volt a.c. light circuit supplies current to the two helical heating coils in the gauge. The two taps on the secondary of this little transformer furnish, respectively, 44.1 milliamperes and 17.8 milliamperes through the $\mathbf{5 1 . 2} \mathrm{ohms}$ of resistance in the two heater coils in series, the voltages being, respectively, 2.25 volts and 0.91 volt and the watts input 0.0993 and 0.0162 watt. We estimate the temperature of the heaters roughly as $52^{\circ}$ and $8.5^{\circ}$ on these two connections. These temperatures we computed from the watts input and the radiating area of the heaters. By means of these two different power inputs we obtain two very convenient scales of pressure for the gauge.

Our method of operating the gauge constitutes an important simplification compared to the methods described by Woodrow or Schrader and Sherwood who went to considerable trouble to maintain constant temperature on the heaters and to measure that temperature by a potentiometer measurement of the voltage drop in the low heater resistance itself. These refinements which were well justified when a research on the gauge was the object are quite unnecessary for a gauge to be applied as a tool to the uses we have in mind. Instead of maintaining the gauge heater at constant temperature for all gas pressures in the gauge we let the heater assume whatever temperature the combined effect of radiation loss and gas conduction loss impose with a constant wattage input to the heater. The small transformer automatically maintains substantially constant wattage on the heater since for the temperature variations in question the nichrome heater wire exhibits no appreciable change in resistance. For very high vacua the temperature of the heater is fixed by the radiation loss alone and is therefore constant for all gas pressures from absolute vacuum up to pressures where the heat loss by gas conduction begins to become appreciable in comparison to the radiation loss. From Eq. (1) it is evident that over this lower range of pressures the deflection of the gauge will be strictly proportional to the pressure. With increasing pressure the cooling effect of the gas conduction away from the heaters will lower the temperature $T_{1}$ in Eq. (1) and the curve of gauge deflection against pressure will fall progressively below the original straight (dotted) line as illustrated in Fig. 4A. Fig. 4B shows the calibration curve of 


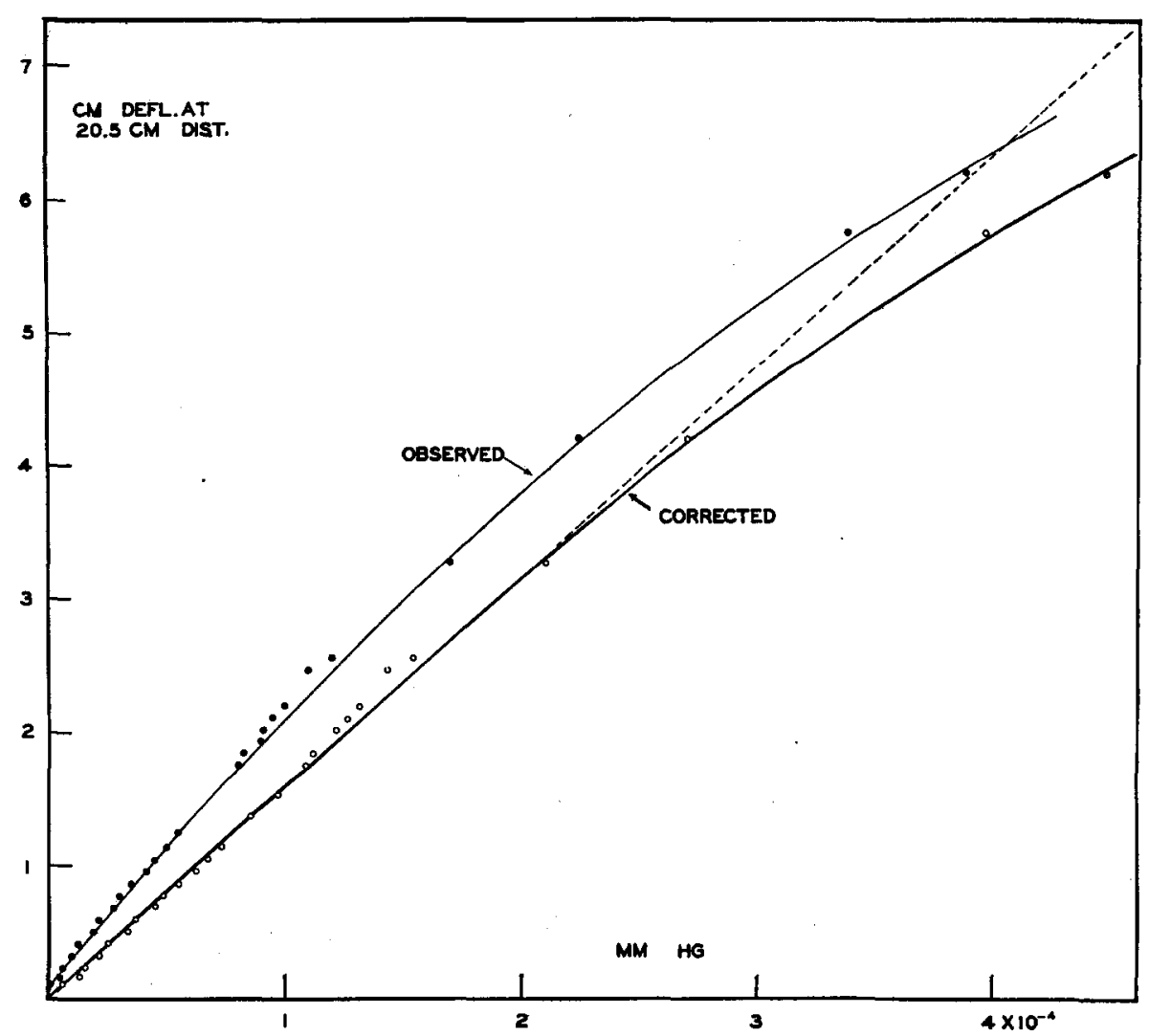

FIG. 4A. Calibration curve of gauge against McLeod readings of pressure for low sensitivity scale.

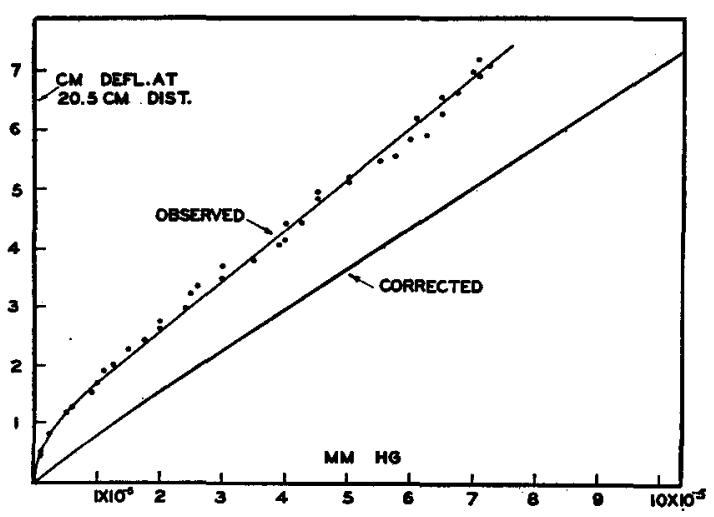

FIG. 4B. Calibration curve of gauge against McLeod readings of pressure for high sensitivity scale.

the gauge for its more sensitive scale (higher temperature and wattage input on heater coils). Fig. 5 illustrates the two scales we obtain with this instrument. The dimensions shown are for a scale only $21 \mathrm{~cm}$ distant from the lens in front of the glass window. It would of course be easy to get many times the sensitivity here shown by running the heater coils at higher wattage input.

Fortunately the nature of the curve represented in Fig. 4A is simple to compute. The deflection $D$ of the gauge away from its "cold zero" position is proportional to the expression (1). Thus

$$
\begin{array}{r}
D=K p\left[\left(T_{1} / T_{0}\right)^{\frac{1}{2}}-1\right] \\
=K p\left\{\left[\left(T_{0}+t\right) / T_{0}\right]^{\frac{1}{2}}-1\right\},
\end{array}
$$

where $K$ is a constant of proportionality depending on the stiffness of the fiber and geometry of the optical system. The watts lost from the heater by radiation may for small temperature rises $t$ above $T_{0}$ be taken proportional to $t$. Let the watts lost by radiation per degree of temperature rise $t$ be $R$. The watts lost by gas conduction will be proportional both to the temperature rise and to the gas pressure in the gauge. At some pressure $P$ which we shall compute below the watts lost by gas conduction for any small 


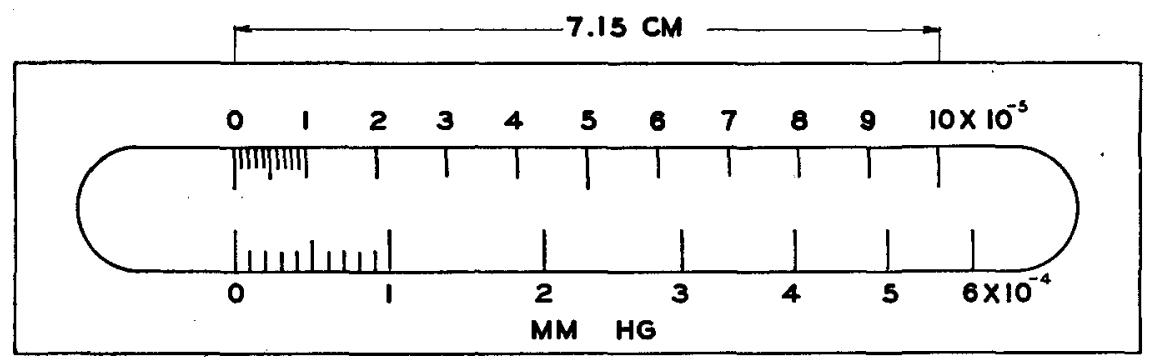

FIG. 5. Actual scale of gauge for a light lever of $20.5 \mathrm{~cm}$.

temperature rise $t$ will just equal the watts lost by radiation $R t$. Then it is evident that the total power loss from the heater will be

$$
W=R t(1+p / P),
$$

where $p$ is the pressure in the gauge. The temperature rise $t$ of the heater will assume such a value that $W$ will just equal the watts supplied to the heater. Expanding the radical in (2) we have with good precision for the low temperature rises $\left(t / T_{0} \ll 1\right)$ in question

$$
D=K p t / 2 T_{0} .
$$

Eliminating $t$ between (3) and (4) we have

$$
D=C p /(1+p / P),
$$

which is the equation of the curve of the gauge deflection $v s$. pressure.

Since this equation involves only two parameters, $C$ and $P$, by measuring the gauge deflection at two suitably chosen known pressures (established in a calibration with a McLeod gauge whose vapor has been shielded from the Knudsen gauge with a liquid-air trap) these constants can be empirically determined and the entire scale is thus smoothly established for the Knudsen gauge. In practice, however, it is safest to observe gauge deflections against McLeed pressures over the entire range to be used as a check because serious errors of the McLeod may be thus revealed. One characteristic error of the McLeod is due to a difference in the capillary depression of the two columns of mercury caused either by a difference in diameter of the two capillaries or by some surface condition of their glass walls. Such a condition displaces the Knudsen deflection vs. McLeod pressure curve throughout most of its length either to the right or left of the true curve and also slightly distorts its shape.

The curves shown in Fig. 4A and B exemplify the necessity for just such a capillary correction. The curve through the observed points in Fig. 4B which was taken with the Knudsen gauge operating on the higher wattage input to its heater coil is seen to be nearly straight over most of its length but not exactly directed toward the origin. Very near the origin however this nearly straight segment is joined to the origin by a rather sharp hook. A precisely similar hook and offset from the origin appears on the other calibration curve, Fig. 4A, for the Knudsen gauge operating on lower wattage input but it is important to notice that the offset and hook occur at very different Knudsen gauge deflections for the two cases but at precisely the same McLeod pressure reading. This circumstance together with the fact that at low pressures as we raised the mercury in the McLeod we noticed that the level in the closed capillary was always slightly in advance of the level in the open capillary led us to conclude that the hook and the offset could not possibly represent a characteristic of the Knudsen gauge and was in all probability the result of a capillary error in the McLeod. The McLeod was read by the familiar method of raising the mercury in the open capillary until it was level with the upper end of the closed capillary and reading the position of the mercury in the closed capillary against a scale calibrated to read pressure directly. This, as is well known, results in a nonuniform scale. A constant capillary depression or elevation of the mercury in the closed capillary relative to the mercury in the open capillary calls then for a nonuniform correction which turns out to be a quadratic function of the observed (erroneous) 
pressure. We found that by assuming a constant capillary elevation of the mercury in the closed capillary amounting to $1.5 \mathrm{~mm}$ we could obtain corrected curves for both Knudsen gauge sensitivities which exhibited no hooks at the bottom end and were directed satisfactorily through the origin. The magnitude of this correction $(1.5 \mathrm{~mm})$ seemed entirely reasonable from the aforementioned behavior of the mercury in the capillaries when the level was being raised for a reading at the lowest pressures. The corrected curves are shown in Fig. $4 \mathrm{~A}$ and $\mathrm{B}$. In $4 \mathrm{~A}$, the curve for less sensitive Knudsen scale, each observed point has been corrected to a new abscissa for the capillary error while in $4 \mathrm{~B}$ the capillary correction has been applied to the smooth curve drawn through the observed points.

Equations for determining the parameters $P$ and $C$ in Eq. (5) from two observed deflections $D_{1}, D_{2}$ at two corresponding pressures $p_{1}, p_{2}$ are

$$
\begin{aligned}
& C=\left(P_{2}^{-1}-P_{1}^{-1}\right) /\left(D_{2}^{-1}-D_{1}^{-1}\right), \\
& P=p_{2}\left(1-D_{2} / D_{1}\right) /\left(D_{2} / D_{1}-p_{2} / P_{1}\right) .
\end{aligned}
$$

From our observed curves the constant $P$ turns out to have the value $1.6 \times 10^{-3} \mathrm{~mm} \mathrm{Hg}$ in the case of the less sensitive scale (low voltage tap on "mouse power" transformer). For the more sensitive scale involving only the low pressures the scale is sensibly linear as one would expect. Most of the errors of observation we feel convinced are due to the uncertainty in reading the McLeod rather than the Knudsen gauge since under conditions where the pressure should not have been changing rapidly the Knudsen gauge read far more consistently the same than did the McLeod.

We have constructed two of these gauges the design being identical in all essential respects in both and we find that the deflections agree admirably when connected to the same vacuum system. We feel therefore that we can safely recommend those who wish to construct a Knudsen gauge but cannot afford the time to make a calibration of their gauge against a McLeod simply to copy the design we have given in this paper and adopt the scales as we show them.

The dimensions of the "mouse power" transformer are given here to assist those who wish to

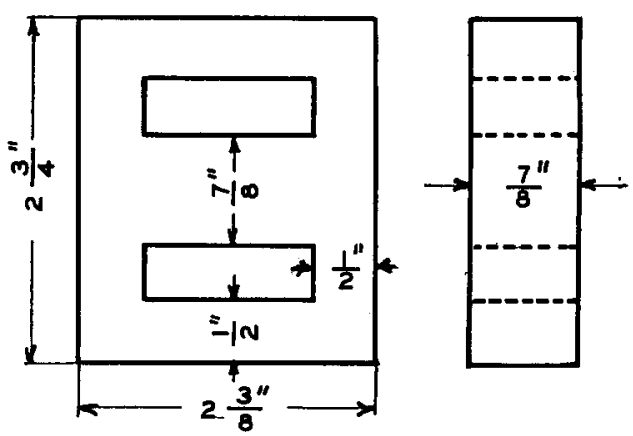

FIG. 6. Core dimensions of "mouse power" transformer.

follow the last-mentioned suggestion. Fig. 6 shows the dimensions of the core laminations. (Any small core with the same or larger cross section of magnetic circuit will do as well.) These are stacked up to a thickness of $7 / 8$ inch. The primary consists of 1000 turns of No. 28 enameled. wire. This is to be connected to 110 volts, 60 cycles a.c. The secondary windings are of number 28 d.c.c. wire. For the more sensitive scale 21 turns are connected to the two heater coils in series and for the less sensitive scale these are connected to 8 turns.

Since we have been pressed for time and the Knudsen gauge was badly needed as a tool in our other work we have not examined its characteristics for gases other than air. From the theory of Knudsen one would expect that the initial slope of the calibration curve should be independent of the nature of the gas save possibly for minor corrections caused by different gases having different thermal accommodation coefficients. With the gauge heaters operated at constant wattage input the curvature of the characteristic should not be entirely independent of the nature of the gas, however, because the watts lost from the heaters by gas conduction will depend on the nature of the gas (varying inversely as the square root of the molecular weight). This will only change the gauge calibration a little at the higher pressures and should be no serious detriment to the use of the gauge for the purposes explained at the beginning of this article.

We take pleasure in acknowledging our grateful indebtedness to Dr. Leon L. Watters of New York City for the funds given as a memorial to his wife, Frances $H$. Watters, which have made possible the entire program of research of which this investigation forms a part. 